\title{
Frontières
}

\section{Les mots de la souffrance}

\section{Jean-Jacques Lavoie}

Volume 17, numéro 2, printemps 2005

Surtout, ne pas souffrir

URI : https://id.erudit.org/iderudit/1073485ar

DOI : https://doi.org/10.7202/1073485ar

Aller au sommaire du numéro

Éditeur(s)

Université du Québec à Montréal

ISSN

1180-3479 (imprimé)

1916-0976 (numérique)

Découvrir la revue

\section{Citer cet article}

Lavoie, J.-J. (2005). Les mots de la souffrance. Frontières, 17(2), 21-27. https://doi.org/10.7202/1073485ar

\section{Résumé de l'article}

Cet article interroge le savoir et les croyances qui sont déposés dans les signifiants qui disent et traduisent la notion de souffrance. L'enquête lexicale est accompagnée d'exemples tirés du livre de Job et des nombreux récits qu'il a suscités dans les trois monothéismes. Ce choix du livre de Job permet de porter un regard critique non seulement sur ce que laissent entrevoir les

considérations philologiques du lexique de la souffrance, mais aussi sur maints discours qui prétendent justifier la souffrance et résoudre son énigme.
Ce document est protégé par la loi sur le droit d'auteur. L'utilisation des services d’Érudit (y compris la reproduction) est assujettie à sa politique d'utilisation que vous pouvez consulter en ligne.

https://apropos.erudit.org/fr/usagers/politique-dutilisation/ 


\section{Résumé}

Cet article interroge le savoir et les croyances qui sont déposés dans les signifiants qui disent et traduisent la notion de souffrance. L'enquête lexicale est accompagnée d'exemples tirés du livre de Job et des nombreux récits qu'il a suscités dans les trois monothéismes. Ce choix du livre de Job permet de porter un regard critique non seulement sur ce que laissent entrevoir les considérations philologiques du lexique de la souffrance, mais aussi sur maints discours qui prétendent justifier la souffrance et résoudre son énigme.

Mot clés: souffrance - livre de Job religion - monothéisme.

\section{Abstract}

This article interrogates the knowledge and beliefs found in the significant factors that express and translate the notion of suffering. The lexical inquiry is supported by examples from the book of Job and the many accounts to which it gave rise in the three monotheisms. The choice of the book of Job allows a critical look not only on the philologic considerations that the reading on suffering permits, but also on several speeches that appear to justify suffering and resolve its enigma.

Keywords: suffering - book of Job religion - monotheism.

\section{LES MOTS DE LA

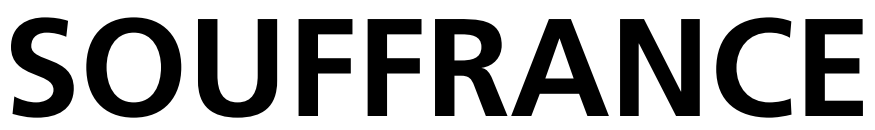

\section{APPROCHE; CONTEMPLE LES MOTS... CHACUN A MILLE VISAGES SECRETS SOUS SON VISAGE NEUTRE}

DRUMOND DE ANDRADE, 1977, P. 76

\author{
Jean-Jacques Lavoie, Ph.D., \\ Département des sciences religieuses, UQAM.
}

Mot: un terme qui vient du latin muttum, qui signifie son, bruit de voix qui n'a pas de signification ou encore grognement d'un animal, particulièrement celui du porc! Comme le terme mutus, muet, il pourrait dériver de mutmut, son à peine distinct, léger murmure. La racine qui serait à l'origine de tous ces termes latins est l'onomatopée grecque $m u$, qui a également donné naissance à certains mots grecs comme muzô, gronder, en parlant du bruit fait par un dauphin, mukaomai, mugir, en parlant du cri des bovidés, muia, mouche, probablement à cause de l'onomatopée $m u$ qui représentait le bourdonnement. Ce parcours, qui va du cri animal à l'impuissance de dire et vice-versa, n'illustre-t-il pas notre propre histoire verbale?

Bien entendu, les mots ne se réduisent pas à des vagissements ou des chuchotements. Particulièrement lorsqu'ils visent à expliquer la souffrance. Ils deviennent alors une monnaie terrible. Surtout entre les mains des philosophes et des théologiens, ces experts qui n'hésitent pas à se payer de mots dans le calme olympien de leurs bibliothèques. Toutefois, mis en face d'êtres souffrants, les philosophes et les théologiens se révèlent tout simplement des faux-monnayeurs. Loin de consoler, leurs discours risquent plutôt d'énerver, d'embêter, voire d'assommer. Dès l'Antiquité, tel était l'avis de Job: il se déclare écrasé et ce n'est pas seulement par ses maux: «Jusqu'à quand m'affligerez-vous et m'écraserez-vous avec des mots?» (Job 19,2). Par contre, les mots que prononce la personne qui souffre doivent être accueillis avec la plus grande attention. Tel est, encore une fois, l'avis de Job: «Écoutez bien mes mots et que ce soit là votre seule consolation!» (Job 21,2). À ses amis, qui sont littéralement des redresseurs de mots (Job 6,26), Job demande non seulement de se taire (Job 13,5), mais il leur rappelle qu'il pourrait très bien faire comme eux et accumuler des mots s'il pouvait échanger leur bien-être contre sa souffrance (Job 16,4).

L'exemple de Job illustre avec éloquence que le seul savoir possible sur la souffrance sauf imposture - est le savoir que la souffrance a d'elle-même. Ce savoir est de l'ordre non de la justification mais du témoignage: «je souffre», «j'ai mal», etc. Ce témoignage est le plus souvent réduit à des balbutiements puisque la souffrance, comme nous le rappelle Job, est l'expérience d'une disproportion: «si mon indignation pouvait être pesée/si on pouvait porter mon affliction sur une balance/ensemble elles seraient plus lourdes que les sables de la mer/c'est pourquoi mes paroles s'étranglent» (Job $6,2-3)$. Indicible parce qu'infini fait chair, la 
souffrance est aussi et avant tout autarcique. C'est d'ailleurs pourquoi le premier discours de Job ne s'adresse à personne: c'est un monologue (Job 3). Qui plus est, les mots de ce monologue, qui font suite à un long silence de sept jours et sept nuits (Job 2,13), sont comparés aux rugissements d'une bête féroce (Job 3,24). Quant aux dialogues qui suivent (Job 4,31), ils nous introduisent dans l'univers du totalitarisme, car «la définition du système totalitaire, c'est que l'écoute n'y existe pas. C'est un régime sourd» (Bellet 1989, p. 41). À l'instar de Job, le mal dont souffrent maintes personnes est aussi fait de «mal-entendus». Ainsi, à la question de savoir comment parler de la souffrance, il n'est qu'une seule réponse: chacun en parle comme il la vit. Toutefois, que la souffrance soit vécue dans la résignation, la plainte ou la révolte, la personne souffrante a besoin de mots pour se dire.

Parmi ces mots, il y a d'abord le mot souffrance et c'est ce mot que je veux analyser dans ce texte, non pas afin de donner une définition du signifié, mais plutôt pour interroger les signifiants qui changent non seulement d'une langue à l'autre, mais aussi à l'intérieur d'une même langue. Mon enquête lexicale, qui ne portera que sur quelques langues qui me sont plus familières, a donc pour but d'apporter un éclairage sur la manière dont la souffrance a été pensée et continue d'être pensée à travers les usages du langage et donc des cultures et des religions. Dit autrement, mon objectif est d'interroger le savoir et les croyances qui sont déposés dans les signifiants qui disent et traduisent la notion de souffrance.

La personne qui souffre n'a pas besoin que de mots. Elle a aussi besoin de récits. Sarah Kofman a bien montré comment les rescapés de la Shoah avaient un besoin intarissable de raconter sans fin, «comme si seul un "entretien infini" était à la mesure du dénuement infini » (Kofman, 1987, p. 16). C'est pourquoi mon étude du vocabulaire de la souffrance sera accompagnée d'exemples tirés du livre de Job et des nombreux récits qu'il a suscités dans les trois monothéismes. J'ai retenu ce livre parce que Job est la figure emblématique du souffrant, figure au miroir de laquelle Juifs, Chrétiens et Musulmans ont sans cesse tenté de penser le problème de la souffrance. En outre, ce choix du livre de Job me permettra de porter un regard critique non seulement sur ce que les considérations philologiques du lexique de la souffrance laissent entrevoir, mais aussi sur maints discours qui prétendent justifier la souffrance et résoudre son énigme.

\section{SOUFFRANCE ET PATIENCE}

Je commencerai mon enquête par le mot qui me sert de fil conducteur: souffrance, ou suffering en anglais, sufrimiento en espagnol,

\section{heure blanche}

berçant une vieille peine

\section{caressant un bonheur perdu}

va se perdre dans le néant

sofrimento en portugais, sofferènza en italien, etc. Ce mot dérive du latin sufferentia qui désigne l'action de supporter; ce mot avait aussi le sens de trêve, de délai ou de répit. Ce sens n'est pas totalement disparu, comme le montre l'expression, peu usuelle il est vrai, «avoir une affaire en souffrance», donc en suspens. Quant au verbe souffrir, qui pourrait aussi être à l'origine du substantif souffrance, il provient du latin populaire sufferire, lequel verbe est une altération du latin classique sufferre, supporter, se soutenir, se maintenir. Ce verbe est composé du préfixe $s u b$ qui marque la position inférieure et du verbe latin ferre, porter, lequel dérive du grec phérô qui a exactement le même sens. Souffrir, c'est donc d'abord porter, supporter, au sens concret de soutenir (un poids). Par extension, le verbe souffrir a aussi pris le sens d'endurer, de patienter, de tolérer. Les deux mots, patience et souffrance, se confondent d'ailleurs dans le terme latin patientia. Encore de nos jours, celui qui souffre et se fait soigner n'est-il pas identifié comme patient ${ }^{1}$ ?

Il n'est donc pas étonnant que souffrance et patience forment un couple inséparable dans maints discours philosophiques et religieux. Par exemple, après tant de souffrances et après avoir enduré les discours moralisateurs de ses visiteurs, Job subira une autre disgrâce: celle de passer à la postérité sous une fausse identité. En effet, la lettre de Jacques $(5,11)$, qui est le seul livre du Nouveau Testament à mentionner Job, après avoir comparé la patience à l'image du cultivateur en attente de la moisson (5,7-9), présente Job comme un modèle de patience ${ }^{2}$ et ne fait allusion qu'à la finale du livre (Job 42,10-17)! Cette interprétation qui défigure le héros révolté en homme patient n'a pourtant aucun fondement dans le texte hébreu du livre de Job. Au contraire, Job fait référence à sa propre impatience (voir, par exemple, Job 21,4), tandis que ses interlocuteurs l'accusent de manquer de patience (Job 4; 15; 18; 34; etc.). Qui plus est, cette lecture tendancieuse proposée dans la lettre de Jacques escamote rien de moins que les chapitres 3 à 31 du livre de Job, c'est-à-dire toute sa révolte! Une véritable conjuration.

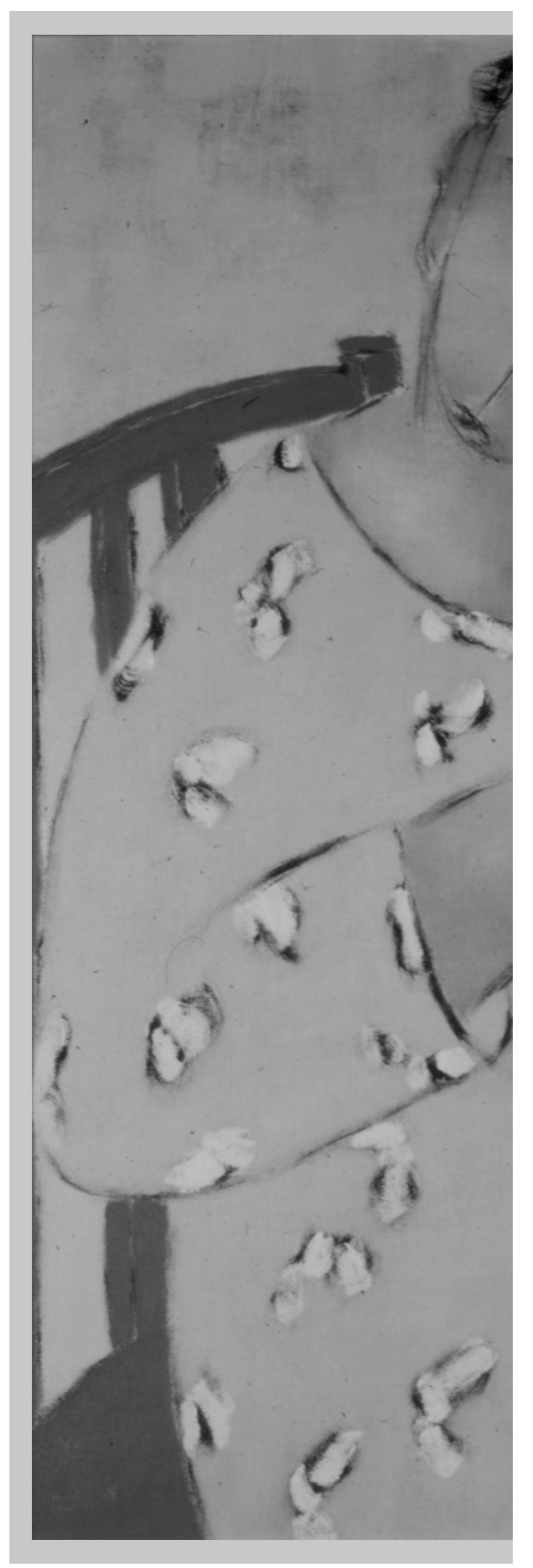

Pourtant, cette lecture abusive connaîtra un grand succès dans la tradition chrétienne. Ainsi, Tertullien présente Job

[...] comme un exemple et un témoignage d'exercice de la patience (patientiae), tant pour ce qui est de l'esprit que pour ce qui est 


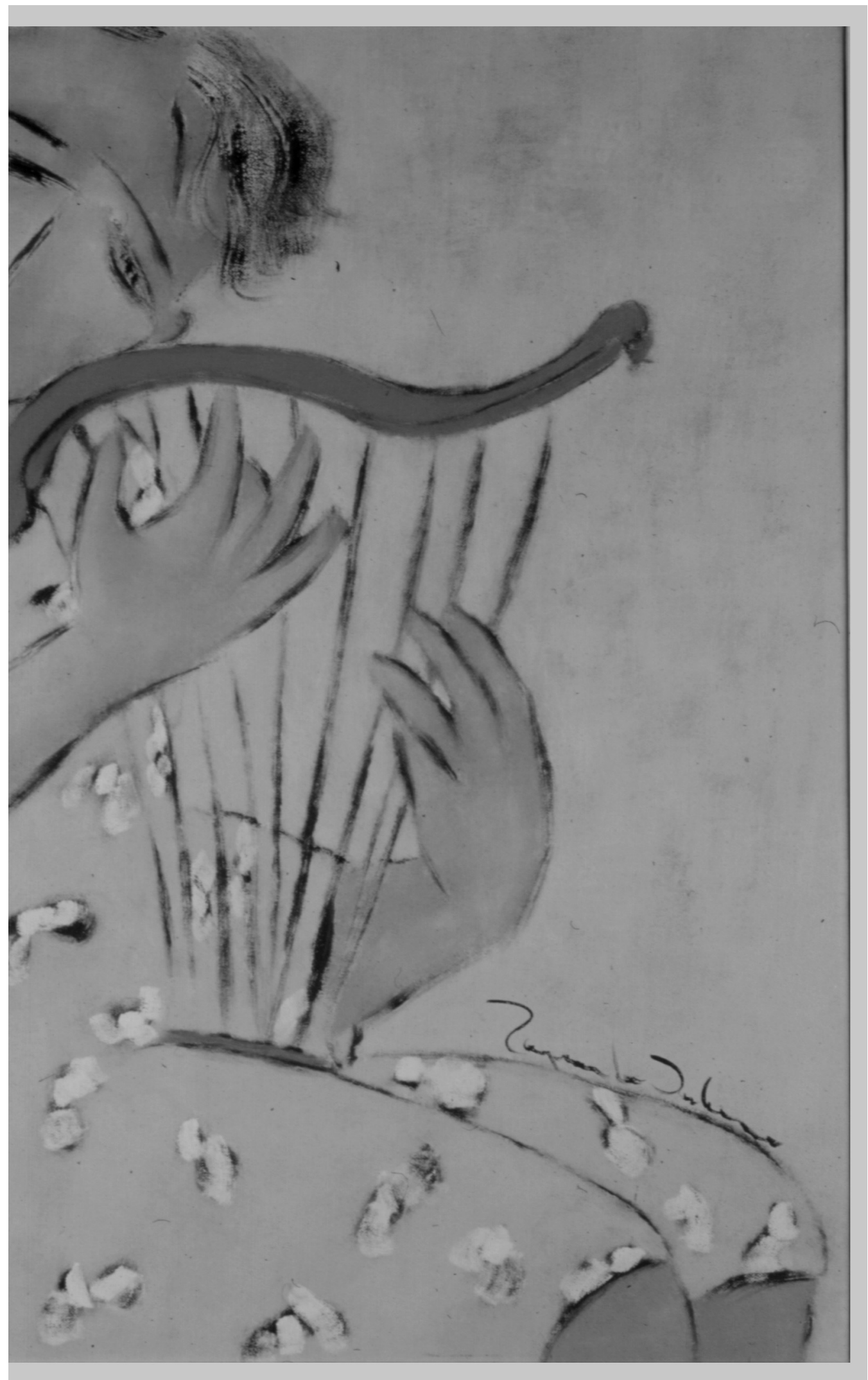

de la chair, tant pour ce qui est de l'âme que pour ce qui est du corps, afin que nous ne soyons abattus ni par la perte des biens du siècle, ni par la disparition des êtres chers, ni non plus par les mauvais traitements infligés à notre corps (Tertullien 14,3). à faire preuve de patience (patientia)!» (Tertullien 14,7). Dans sa théologie de la souffrance, Jean Chrysostome adopte lui aussi la solution philosophique du stoïcisme (Nowak, 1972, p. 88, 225-226). Cette fameuse patience de Job est positivement légendaire dans la tradition chrétienne. $\mathrm{Au} \mathrm{XV}$ siècle, Le Mystère de la patience de $J o b$ est un récit très célèbre (Meiller, 1971). Fidèle à une tradition plus que millénaire, deux siècles plus tard, Fénélon est d'avis que «seuls les hommes indociles et corrompus s'étonnent de ce qu'on leur propose pour modèle de patience Job» (Leduc-Fayette 1996, p. 68).

Bien entendu, au cours des siècles, Job n'a pas été le seul modèle de patience proposé aux chrétiens. Ces derniers ont aussi été invités à trouver patience et repentir dans la contemplation du Christ souffrant en croix. Par ailleurs, cette mystique de la croix, plus ou moins imprégnée de dolorisme selon les époques, s'est très souvent transformée en une justification de la souffrance elle-même, voire en une survalorisation de la souffrance comme prolongement de la Passion, le Christ n'étant plus qu'un autre résigné exemplaire. Bref, peu importe le modèle choisi, maints chrétiens ont identifié et identifient toujours la patience avec la résignation et l'impatience avec le péché et la révolte.

Ce portrait d'un Job patient présenté par la lettre de Jacques et les écrits de ses successeurs chrétiens avait déjà une solide base textuelle dans le monde juif. En effet, la traduction grecque du livre de Job avait non seulement déjà réduit le texte d'environ un sixième, mais elle avait en outre atténué les attaques de Job contre Dieu et imaginé un Job patient et résigné (Ziegler, 1982). Le Testament de Job, œuvre juive datant autour de l'an 50 avant notre ère, ignore non seulement la révolte de Job, mais le décrit de manière stoïcienne comme un athlète (Testament de Job 4,10; 27,3-5) qui invite ses enfants «à faire preuve de patience [...], car la patience triomphe de tout» (Testament de Job 27,7). Dans la tradition rabbinique, incapable d'occulter l'impatience de Job, on la lui reproche (Talmud de Babylone, Semahôt 8) et on associe le rebelle à la génération du déluge, à l'Égypte, à Gog et Magog et aux méchants qui sont condamnés douze mois à la géhenne (Talmud de Babylone, 'Eduyôt 2,10). Cette condamnation de Job est réitérée dans la tradition mystique (Zohar 2,33b). Plus près de nous, en hébreu moderne, le nouveau terme qui a été forgé pour dire souffrance, sivelâh, a un dérivé, savlanût, qui signifie patience. Ces deux néologismes dérivent de la racine svl qui, en hébreu biblique et talmudique, signifie porter, être chargé; cette racine est aussi à l'origine du mot sévèl, fardeau, 
charge. L'hébreu moderne nous confirme ainsi que souffrance et patience sont indissociables; il faut donc être patient dans la souffrance, il faut savoir porter son fardeau. Le rabbin Solomon Schechter ne dit pas autre chose: "Celui qui souffre doit accepter sa souffrance en priant dans un esprit de soumission; il doit reconnaître qu'il méritait le châtiment (visitation) divin. » (Schechter 1966, p. 209.)

De son côté, le Coran ne connaît qu'un Job patient: "Nous l'avons trouvé patient (saber) - Quel excellent serviteur! Il était plein de repentir!»(Coran 38,44). Cette patience-soumission, qui s'oppose à la plainte (Coran 14,21) et qui permet de supporter les arrêts divins (voir aussi Coran 52,$48 ; 68,48 ; 76,24$; etc.), constitue une vertu majeure de l'islam et c'est pourquoi le musulman doit prier pour l'obtenir (Coran 2,45). Qui plus est, dans les commentaires du Coran, «le concept de $\underline{s} a b r$, dans toutes ses nuances de sens, est essentiellement hellénistique, car il contient l'ataraxia stoïcienne et la patience chrétienne, la mâ̂trise de soi et le renoncement ascétique» (Wensinck, 1995, p. 705). C'est ce qu'illustrent très bien les diverses traditions musulmanes qui soulignent avec emphase la soumission et la résignation de Job. Par exemple, selon la tradition de Hasan al-Basri, chaque coup qui frappe Job est une occasion pour lui de prononcer un discours ascétique (Déclais, 1996, p. 188-191). Selon Ibn 'Asâkir et al Kisâ‘î, des gestes concrets traduisent la patience de Job: «Lorsqu'un vers tombait de son corps, il le prenait et le remettait à sa place en disant: "mange la bénédiction de Dieu” » (Legrain, 1985, p. 61-62; voir déjà le Testament de Job 20,8-9). Al-Baydâwî est d'avis que c'est Job lui-même qui «aurait demandé une mise à l'épreuve de sa patience» (Legrain, 1985, p. 61). Selon Ibn 'Asâkir, la patiencerésignation de Job est exemplaire non seulement pour tous les musulmans, mais aussi pour tous les vivants: "Mémorial de patience pour les vivants, Job sera à la tête des patients au jour de la résurrection (Legrain, 1985, p. 70). Maints commentateurs musulmans affirment aussi que c'est par Job que Dieu, lors du jugement dernier, confondra les malades qui auront été impatients (Legrain, 1985, p. 70). Cet idéal d'impassibilité (apatheia), qui était déjà promu par les sages stoïciens, est également bien présent dans les hadiths qui rappellent la sévérité de Muhammad à l'égard des rites de lamentation lors des deuils. Le hadith suivant résume bien la volonté du Prophète à ce sujet: "Selon Anas ben Mâlek, le Prophète (à lui bénédiction et salut), passant devant une femme qui pleurait sur une tombe, lui dit: "Crains Dieu et résigne-toi" » (El-Bokhâri 80, 1991, p. 159). Dans les

UNE PATIENCE QUI NE SAIT PAS S'INDIGNER EST UNE FAUSSE PATIENCE, UNE PASSIVITÉ OU UNE AUTO-CONDAMNATION QUI N'OSE PAS S'AVOUER, UN FATALISME QUI A OUBLIÉ POURQUOI IL PATIENTE ET CONTRE QUOI IL PATIENTE.

hadiths, la notion de patience se confond à maintes autres reprises avec celle de résignation. Toujours selon El-Bokhâri, cette patience-résignation est même une grande vertu: «Celui qui montre vouloir supporter les choses avec résignation, Dieu lui donnera de la résignation. Personne n'est gratifié d'un don meilleur, ni plus opulent que la résignation.» (Bokhâri 77, 1991, p. 159.)

Force est de constater que les trois monothéismes ont plus ou moins valorisé, selon les époques, la patience-résignation, la patience-passivité, ce dernier mot dérivant, lui aussi, du latin patior, souffrir, pâtir, passivement. Toutefois, ce lien inséparable entre souffrir et patienter peut-il être compris autrement qu'une résignation stoïque ou bigote? À mon avis, non seulement il peut être compris autrement, mais il le doit. En effet, l'association entre la patience et le simple fait de savoir attendre est superficielle. La véritable patience n'a rien à voir avec l'attentisme et la passivité. Une telle patience n'est que tyrannie et négation morbide des désirs. C'est d'ailleurs pourquoi les régimes totalitaires ont toujours su valoriser une telle patience. La véritable patience n'a pas plus de lien avec une conception religieuse de la vie humaine qui ne serait qu'attente d'un paradis quelconque. Une telle patience n'est qu' «au-delâtrie» ou «idolâtrie de l'au-delà ». La véritable patience n'est pas davantage une simple résignation ${ }^{3}$. Elle est même tout le contraire. Elle est une action: si souffrir est supporter, patienter est résister, lutter. Une patience qui ne sait pas s'indigner est une fausse patience, une passivité ou une auto-condamnation qui n'ose pas s'avouer, un fatalisme ${ }^{4}$ qui a oublié pourquoi il patiente et contre quoi il patiente. Or, celui qui souffre doit d'autant plus se rappeler contre quoi il patiente et résiste que sa souffrance lui donne le sentiment que le temps s'immobilise dans un présent absolu, un présent sans ouverture et sans échéances. En effet, la souffrance est ressentie non seulement comme une réalité insupportable, mais aussi comme une réalité sans fin. C'est bien ainsi que Job nous décrit sa souffrance (Job 6,8-10; 7,3-4; 16,15-16; etc.). Certes, celui qui souffre est capable d'anticipation, mais l'exercice d'une telle capacité ne libère en rien du présent; au contraire, il en augmente l'intensité (Job 3,21-26; 6,11-13; 19,8-10; etc.). Ce sentiment d'un arrêt du temps, d'un présent figé et immobile est si étouffant qu'il arrive même que la mort soit moins pire que la souffrance. Tel est le cas de Job pour qui la mort devient par moment le seul espoir (Job 3,11.13.20; 7,15; 10,18-19; etc.). Quand la souffrance devient plus terrifiante que la mort, souffrir est alors endurer le non-endurable. Certes, endurer est encore espérer, même si le seul espoir qui reste est la mort, mais force est, dès lors, de constater que cette souffrance plus terrifiante que la mort peut être une des limites qui s'impose à la patience. Ainsi, même la patience comprise positivement comme acte de résistance doit avoir des limites déterminées par les circonstances. Ces limites, qui sont différentes pour chacun, sont essentielles, car elles évitent les excès ou les dérives vers la fausse patience qui n'est que passivité morbide, résignation stoïque et fatalisme. En définitive, ces limites indiquent que la patience, qui consiste à renoncer au renoncement, ne s'oppose pas vraiment à l'impatience jobienne, impatience nourrie d'indignation, d'espérance, de ténacité et de désir de l'Autre. Patience et impatience sont plutôt deux vertus complémentaires qui s'actualisent dans une même lutte contre la souffrance.

\section{SOUFFRANCE ET CHÂTIMENT}

La souffrance est complexe, car elle constitue un des éléments du problème du mal, qu'il soit physique (douleur ou souffrance), moral (volonté maligne, faute) ou métaphysique (finitude). En effet, le mal véhicule de la souffrance avec lui. C'est peut-être pourquoi on dit «j'ai mal» lorsqu'on souffre. D'ailleurs le mot malade, anciennement prononcé malabde, dérive lui-même de l'adverbe latin male, qui est associé à l'origine au mot mal, et du verbe habere, être ou se trouver dans tel ou tel état. La maladie serait donc un mauvais état. À moins que ce ne soient les mauvaises habitudes qui conduisent à la maladie! Par ailleurs, la distinction linguistique entre souffrance et mal est déjà un premier indice qui signale que la souffrance n'est pas un simple synonyme du mal. On ne peut donc rattacher à tout prix la souffrance au mal, surtout au mal moral. Ce serait là réduire toute forme de souffrance à une souffranceculpabilité ou, pire, à une souffrancechâtiment. Or, force est de constater que s'il peut y avoir de la souffrance-coupable, 


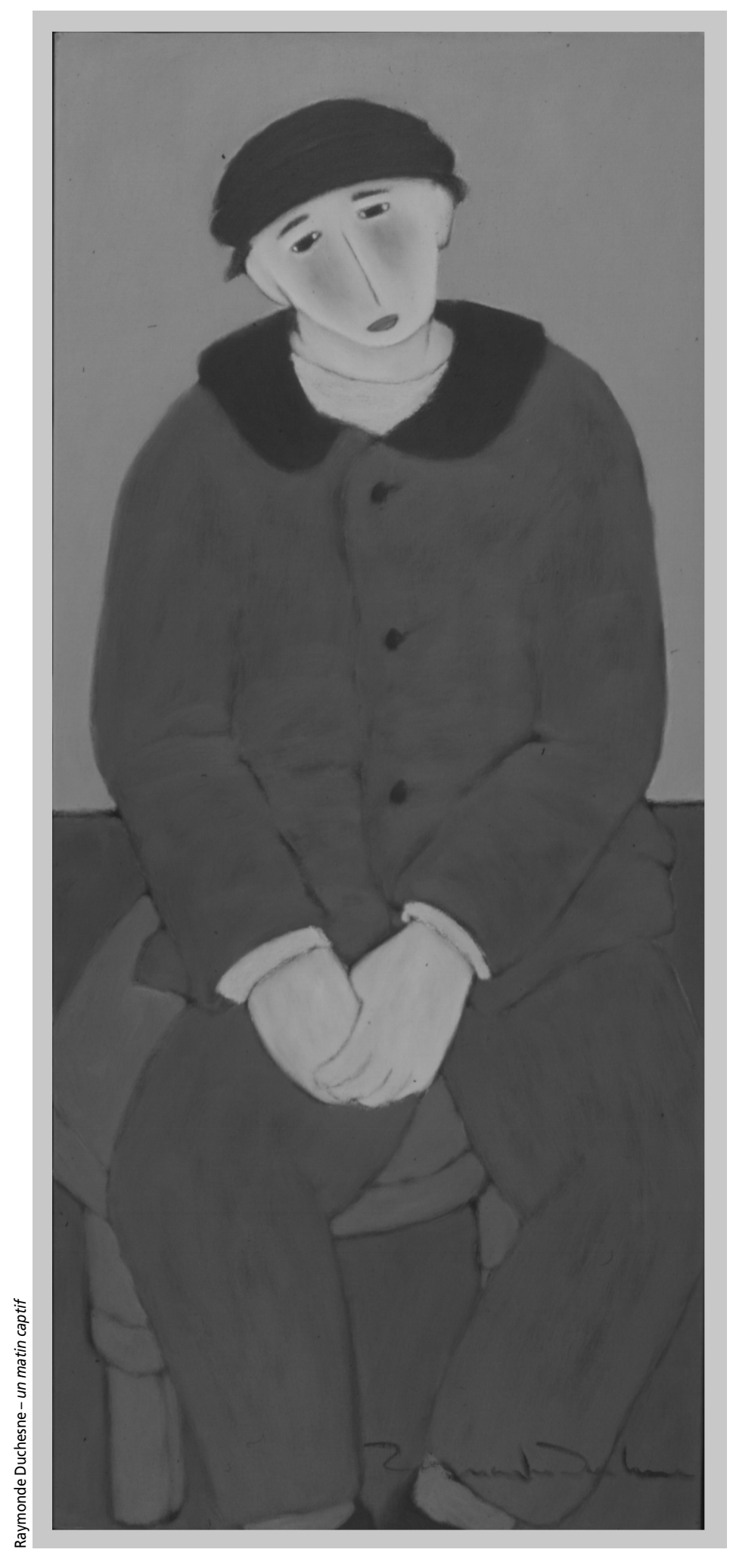

du mal-coupable, il y a aussi de la souffrance-malheur, du malmalheur ${ }^{5}$.

L'analyse philologique du lexique de la souffrance laisse néanmoins entrevoir une histoire de la pensée où les liens entre souffrance-culpabilité et souffrance-châtiment sont très solides. C'est ce qu'on peut déduire de l'anglais pain, qui peut signifier douleur, souffrance ou peine, punition. Ce mot dérive du latin poena, peine, douleur ou punition, châtiment; quant au mot latin, il provient du terme grec poinè, expiation, châtiment. Enfin, le terme grec dérive lui-même d'une racine sanskrite $p u$ qui renvoie

\section{J'ai eu une envie de suivre la nuit et $y$ dormir à jamais}

à l'idée de purification. Ainsi, l'étymologie nous apprend que la souffrance peut être considérée comme un châtiment, une punition ou une purification.

Ce lien entre souffrance et châtiment n'est pas propre au vocabulaire anglais et latin. On le retrouve aussi dans le verbe grec paschô. Le premier sens de ce verbe est celui de recevoir une impression ou une sensation. Lorsque le sentiment ressenti est mauvais, le verbe prend le sens de souffrir, mais aussi d'endurer, voire d'être châtié. Cette perception de la souffrance est donc non seulement associée à la patience, comme dans le mot grec hupomonè, mais aussi à celle de châtiment. Si la souffrance est un châtiment, c'est qu'elle est la conséquence d'une faute morale, d'une culpabilité.

De manière similaire, l'arabe 'adhab signifie aussi bien douleur que châtiment. Quant au terme que dispose l'hébreu rabbinique pour désigner la souffrance, il a lui aussi une connotation morale: yissurîm signifie aussi bien souffrance que châtiment, puisqu'il dérive du verbe yâsar, châtier, corriger. On retrouve la même ambivalence ou la même vision synthétique de la vie dans le mot hébreu 'âwôn qui signifie, selon les contextes, faute, crime ou peine, châtiment. Enfin, comme dernier exemple, le mot allemand Leid, souffrance, peine, et le verbe leiden, souffrir, avoir mal, dérivent de la famille de mots grecs en aleit-/alit- qui comportent l'idée de faute: aleitès, celui qui est en faute, alitein, commettre une faute à l'égard de, alitèmon et alitros, coupable, criminel.

Ces associations entre souffrance-châtiment, souffrancepurification et souffrance-faute, que laissent entrevoir maintes langues, se retrouvent explicitement dans les chapitres 3 à 37 du livre de Job (Lavoie, 2004, p. 707-755). Par exemple, Job est censé reconnaître sa propre culpabilité lorsqu'il entend ses amis reprendre les mêmes mots qu'il utilisait pour décrire sa maladie, mais cette fois-ci pour décrire la maladie dont souffre le méchant puni par Dieu (Job 8,19a; 15,22a.23b; 18,13.18; etc.). Quant à la souffrance de Job, elle est aussi perçue par ses amis comme une cure médicinale et une correction divine (Job 22,4a; 36,7-12.15-16.21; etc.). Bref, pour les amis de Job, qui ne sont à ses yeux que des «guérisseurs de néant» (Job 13,4), les discours sur la faute ou le péché sont par eux-mêmes et en premier lieu des discours sur la souffrance.

Ces «guérisseurs de néant» auront de nombreux épigones dans les traditions monothéistes. Ainsi, la tradition rabbinique, qui fait toujours autorité dans le monde juif, propose de distinguer entre deux sortes de souffrance: celle qui n'est pas due au péché, qui est souffrance par amour (yesûrîm shèl 'ahavâh), et celle due au péché, qui n'est pas souffrance par amour, mais simple souffrance-châtiment (yisûrîm). Comme l'indique le texte qui suit, la souffrance par amour se reconnaît par le fait qu'elle n'empêche pas la prière ou l'étude de la Tora:

Si un homme est visité par la souffrance, qu'il examine ses actes, selon le verset: Examinons et éprouvons nos comportements, et retournons à Yhwh! (Lamentations 3,40). Si, étant examiné, il ne trouve pas de faute, qu'il attribue son malheur à la négligence de l'étude de la Tora, ce que suggèrent les mots: Heureux l'homme que tu châties, Yah, et que tu instruis par ta Tora (Psaume 94,12). S'il fait cela et qu'il ne trouve toujours pas de faute, il s'agit sûrement de 
DANS UN ÉTRANGE RAPPORT

\section{AU POINT QU'ILS OUVRENT SUR LE MÊME HORIZON.}

souffrance par amour, comme il est dit: Yhwh réprimande celui qu'il aime

(Proverbes 3,12).

(Talmud de Babylone, Berakôt 5a.)

Certains sages, sans attendre d'être visités par la souffrance pour racheter leurs éventuelles fautes, accomplirent des actes de mortification en vue d'atteindre l'union mystique avec Dieu. C'est le cas de Rabbi Hiyya bar Ashi qui pratiqua une abstinence rigoureuse et jeûna si longtemps qu'il finit par en mourir (Talmud de Babylone, Qiddushin 81b). Par ailleurs, cette tendance à l'ascétisme et au dolorisme est loin de faire l'unanimité dans le judaïsme, voire dans le Talmud lui-même qui affirme également que les souffrances ne sont nullement nécessaires pour racheter les fautes. C'est ce que montre la prière de Rava: «Les péchés que j'ai commis, efface-les par ton abondante miséricorde, et non au moyen de souffrances et de maladies». (Talmud de Babylone, Berakôt 17a.)

Cette ambivalence à l'égard de la souffrance est présente dans maints autres textes talmudiques. Par exemple, malgré le fait que Rabbi Yohanan ait repris à son compte la célèbre maxime de Rabbi Aqiba, à savoir que "Précieuse est la souffrance» (Talmud de Babylone, Berakôt 5a), voici ce qu'il répondit lorsqu'on lui demanda durant une maladie s'il accueillait de bon gré les souffrances: "Ni elles, ni les récompenses qu'elles promettent. » (Talmud de Babylone, Berakôt 5b.) Par cette réponse, le sage indiquait non seulement que la maxime d'Aqiba ne pouvait se mettre en pratique, mais il critiquait aussi l'acceptation trop enthousiaste de la souffrance de certains de ses coreligionnaires.

Depuis les origines, maints chrétiens ont présenté la souffrance comme un châtiment et comme une intervention pédagogique de Dieu. Les exemples abondent non seulement dans la littérature d'édification, mais aussi dans les ouvrages théologiques prétendument sérieux (voir à ce sujet de nombreuses références dans Lavoie, 1994, p. 157-158). Tout récemment encore, le tristement célèbre télé-évangéliste états-unien Pat Robertson, tout en s'acharnant contre les musulmans, s'est « réjoui des attentats du 11 septembre, sanction divine imposée à un pays coupable d'avoir toléré avortement et homosexualité»(Warde, 2002, p. 10).

Du côté de l'islam, toute souffrance vient directement ou indirectement de Dieu et a également valeur soit de châtiment, soit d'utilité pour celui-là même qui souffre (Brunschvig, 1974, p. 74-83; Gardet, 1967, p. 96-97). Le hadith suivant illustre bien cette croyance:

Yahya-ben Ya'mar rapporte que 'Aïcha a dit: "Comme j'interrogeais l'Envoyé de Dieu au sujet de la peste, il m'informa que c'était un châtiment que Dieu envoyait contre qui il lui plaisait, mais qu'il en avait fait un moyen de clémence pour les Croyants. Celui qui sera atteint par la peste, alors qu'il est resté dans son pays, résigné et confiant, sachant que Dieu ne frappe que ceux qu'il a prédestinés, aura une récompense égale à celle d'un martyr.» (El-Bhokâri 50,10, 1977, p. 531.)

Sans étonnement, on retrouve aussi la même association entre péché et souffrance. Le hadith suivant est un exemple éloquent parmi plusieurs autres: "Tout ce qui atteint le Musulman, épuisement, maladies, chagrins, douleurs, souffrances, angoisses, même une simple piqûre d'épine lui vaut de la part de Dieu la rémission d'une partie de ses péchés.» (El-Bokhâri 123, 1991, p. 251.)

Malgré ces nombreuses interprétations religieuses de la souffrance, qui ont en outre donné libre cours à des spiritualités doloristes, voire à des comportements sadomasochistes, surtout dans le catholicisme, mais aussi dans le shi'isme (Ayoub, 1978) et dans certaines traditions du judaïsme comme le qaraïsme et la kabbale (Vajda, 1989, p. 31-43), il est remarquable de noter que, dans la finale du livre de Job, Dieu donne tort aux amis de Job qui n'ont cessé de percevoir sa souffrance comme un châtiment ou une épreuve purificatrice (Job 42,6). Il leur donne tort parce que la logique éthique de leurs théodicées, qui n'arrive pas à penser la souffrance autrement qu'en termes de culpabilité, est une source d'immoralité. C'est en effet à cause de leur langage éthique sur la souffrance que ces piètres avocats de Dieu ont pu se maintenir à distance de Job. En effet, s'ils se sont interdits de lutter contre la souffrance de Job, c'est que celle-ci était perçue comme une souffrance purificatrice, une souffrance-châtiment, donc une souffrancecoupable et voulue par Dieu. En réfutant ces associations souffrance-châtiment, souffrance-purification et souffrance-faute, Dieu reconnaît donc que la morale ne peut être employée comme avenue épistémolo- gique à l'intérieur d'un discours religieux sur la souffrance. Bref, le Dieu du livre de Job refuse de se laisser enfermer dans la logique éthique qui sous-tend maints mots et discours anciens et contemporains.

En Job 42,6, Dieu ne se contente pas de donner tort aux amis de Job. Il donne aussi raison à Job, montrant ainsi que les questions soulevées par ce révolté sont pertinentes et que la foi elle-même doit les assumer. Ainsi, une théologie qui n'est pas embarrassée par la souffrance inintelligible et ténébreuse est certainement une théologie superficielle et peu crédible (Lavoie, 1994, p. 160-164). Qui plus est, une théologie comme celle des amis de Job, qui se contente d'expliquer théoriquement la souffrance sans même chercher à la combattre concrètement, est une théologie qui ignore que sa tâche première est de marier les mots à la chair, une théologie désincarnée, froide et abstraite qui ne peut que susciter soit de l'indifférence, soit du dégoût.

\section{POUR NE PAS CONCLURE...}

«Oui, beaucoup de philosophie,

beaucoup de chagrin et qui accroît la

connaissance accroît la souffrance.»

(Qohélet 1,18).

Après des millénaires de réflexions, cette déclaration de Qohélet n'est toujours pas démentie: le savoir apporte une souffrance spécifique $^{6}$. Il aiguise la douleur. Y aurait-il donc un bonheur enviable de l'innocence, de l'ignorance, voire de la paresse? Faut-il donc rejeter les consolations que pourraient apporter les mots issus de la plume d'un philosophe ou d'un théologien? Je laisse le soin à chacun de répondre à ces questions. Cependant, peu importent les réponses qu'on y apportera, tout philosophe ou théologien le moindrement honnête reconnaîtra que l'aporie de la raison conditionne de façon fondamentale sa foi, que celle-ci soit religieuse ou athée.

Le sage Qohélet reconnaissait déjà que la souffrance est le prix de la raison et que penser et souffrir sont intimement liés dans un étrange rapport au point qu'ils ouvrent sur le même horizon. La sagesse a donc à voir avec le deuil ${ }^{7}$ d'une connaissance totale et de la quête d'un sens à tout prix. Face à la souffrance, la sagesse nous rappelle qu'un travail de deuil est nécessaire, car seul un tel travail permet de consentir à la mise en échec de la pensée spéculative par la souffrance, laissant ainsi les vagissements, les chuchotements et les lamentations se dire, même comme rébellion contre Dieu. C'est d'ailleurs à ce travail de deuil que nous invite le livre de Job. Un travail de deuil de Dieu, puisque «tout deuil est d'abord deuil de Dieu»(Jabès, 1973, p. 84). Puis, un travail de deuil avec Dieu ou contre 
Dieu, mais jamais sans Dieu. En effet, la souffrance de Job reste toujours une souffrance de l'Autre, une attente impatiente de l'Autre. N'est-ce pas le seul «avantage»mais combien tragique - du croyant sur l'incroyant que d'avoir un interlocuteur sur qui déverser toute sa plainte et sa révolte? Autrement, et puisqu'il est question ici de mots, ne serait-il pas intellectuellement beaucoup plus simple de rayer le mot Dieu de son vocabulaire?

\section{BIBLIOGRAPHIE}

AYOUB, Mahmoud (1978). Redemptive Suffering in Islam. A Study of the Devotional Aspects of 'Ashura' in Twelver Shi'ism, La Haye, Mouton.

BELLET, Maurice (1989). L'écoute, Paris, Desclée de Brouwer.

BIBLIA HEBRAICA STUTTGARTENSIA (1977). Sous la direction de K. Elliger et W. Rudolph, Stuttgart, Deutsche Bibelgesellschaft.

BRUNSCHVIG, Robert (1974). "Analyse mu'tazilite de la douleur», Mélanges d'islamologie, Leiden, Brill, p. 74-83.

CHALIER, Catherine (2003). Traité des larmes. Fragilité de Dieu, fragilité de l'âme, Paris, Albin Michel.

DÉCLAIS, Jean-Louis (1996). Les premiers musulmans face à la tradition biblique. Trois récits sur Job, Paris, L'Harmattan.

DRUMMOND DE ANDRADE, Carlos (1977). Reunia, Rio de Janeiro, Jose Olympio.

EL-BOKHÂRI (1977). Les traditions islamiques, traduites de l'arabe avec notes et index par O. Houdas, Tome 2, Paris, Librairie d'Amérique et d'Orient \& maisonneuve.

EL-BOKHÂRI (1991). L'authentique tradition musulmane, traduction, introduction et notes par G.H. Bousquet, Paris, Sinbad.

FREITHEIM, Terence E. (1989). The Suffering of God. An Old Testament Perspective, Philadelphia, Fortress Press.

GARDET, Louis (1967). Dieu et la destinée de l'homme, Paris, J. Vrin.

JABÈS, Edmond (1973). El, ou le dernier livre, Paris, Gallimard.

JONAS, Hans (1987). "The Concept of God after Auschwitz: A Jewish Voice», The Journal of Religion, 67, p. 1-13.

KITAMORI, K. (1965). Theology of the Pain of God, Richmond, John Knox.

KOFMAN, Sarah (1987). Paroles suffoquées, Paris, Galilée.

LAVOIE, Jean-Jacques (1994). «Job au-delà des versets: une théologie critique de la souffrance après Auschwitz», dans C. Ménard et F. Villeneuve (dir.), Dire Dieu aujourd'hui, Montréal, Fides, p. 147-172.

LAVOIE, Jean-Jacques (2004). "Job», La Bible expliquée, Villiers-le-Bel, Société biblique française, p. 707-755.

LEDUC-FAYETTE, Denise (1996). Pascal et le mystère du mal. La clef de Job, Paris, Cerf.
LEGRAIN, Jean-François (1985). «Variations musulmanes sur le thème de Job ", Bulletin d'études orientales, 37, p. 51-90.

MEILLER, A. (1971). Le Mystère de la patience de Job, Paris, Klincksieck.

MOLTMANN, Jürgen (1974). Le Dieu crucifié, Paris, Cerf.

NOWAK, Edward (1972). Le chrétien devant la souffrance. Étude sur la pensée de Jean Chrysostome, Paris, Beauchesne.

POHIER, Jacques (1985). Dieu fractures, Paris, Seuil.

SCHECHTER, Salomon (1966). La pensée religieuse d'Israël, Paris, Éditions universitaires.

TALMUD DE BABYLONE (1933). Texte hébreu édité par Lazarus Von Goldschmidt Der Babylonischer Talmud, La Haye, Nijoff.

TERTULLIEN (1984). De la patience, introduction, texte critique, traduction et commentaire par Jean-Claude Fredouille, Paris, Cerf.

TESTAMENT DE JOB (1987). La Bible. Écrits intertestamentaires, traduit, présenté et annoté par Marc Philonenko, Paris, Gallimard, p. 1603-1645.

THE GREEK NEW TESTAMENT (1975). Sous la direction de Kurt Aland et al. New York et Londres, United Bible Societies.

THE HOLY QUR'AN. Arabic Text and English Translation with a Commentary, Edited by Malik Ghulam Farid, Londres, Islam International Publications LTD.

VAJDA, Georges (1989). Sages et penseurs sépharades de Bagdad à Cordoue, Paris, Cerf.

VARILLON, François (1975). La souffrance de Dieu, Paris, Le Centurion.

WARDE, Ibrahim (septembre 2002). "Il ne peut y avoir de paix avant l'avènement du Messie" ", Le Monde diplomatique, $\mathrm{n}^{\circ}$ 582, $49^{\mathrm{e}}$ année.

WENSINCK, A.J. (1995). "Sabr», Encyclopédie de l'islam, Tome 8, Leiden, Brill, p. 705-707.

ZIEGLER, Joseph (1982). Septuaginta: Vetus Testamentum graecum auctoritate academiae scientiarum Gottingensis editum, Job, vol. 12.4, Göttingen, Vandenhoeck \& Ruprecht.

ZOHAR (1975). Sepher ha-zohar (Le livre de la splendeur), tome 3, traduit par Jean de Pauly, Paris, Maisonneuve et Larose.

\section{Notes}

1. Dans nos milieux de soins, celui qui souffre reçoit aussi d'autres noms évocateurs. Par exemple, celui de «client» qui ne souligne que l'aspect pécuniaire de la relation, et celui de «cas» qui ne retient que l'aspect pathologique. Dans ces deux nominations, le sujet est pour ainsi dire disparu au profit d'une catégorie économique et nosographique.

2. Patience est alors la traduction du grec hupomonè, un dérivé du verbe hupoménô, qui signifie littéralement rester sous, d'où soutenir, supporter, rester en place, mais aussi attendre, consentir, accepter, se résigner, voire souffrir. Souffrir et patienter forment donc une fois de plus un couple indissociable.
3. La valorisation d'une telle patience-résignation a probablement une origine théologique. Dieu étant omnipotent, autoritaire et inébranlable, le croyant n'a qu'à se résigner et à s'écraser. Or, à regarder l'histoire de l'humanité, force est de constater que la toute-puissance de Dieu ne va pas de soi. C'est d'ailleurs pourquoi, depuis l'Antiquité jusqu'à nos jours, la réponse à la question posée par l'inefficacité de Dieu face à la souffrance n'a guère varié. $\mathrm{Ou}$ Dieu ne peut rien faire contre la souffrance, mais alors est-il vraiment tout-puissant? $\mathrm{Ou}$ il ne veut pas, mais alors est-il encore bon? Ou encore il peut et veut, mais pourquoi alors y a-t-il autant de souffrance injustifiée dans le monde? Il n'est donc pas surprenant que cette prétendue toute-puissance de Dieu ait été et reste encore pour plusieurs le roc de l'athéisme. Depuis la Shoa, ce sont d'ailleurs des objections à certaines conceptions de la toute-puissance divine qui ont incité maints penseurs juifs (Chalier, 2003; Jonas, 1987) et chrétiens (Kitamori, 1965; Moltmann, 1974; Varillon, 1975; Pohier, 1985; Freitheim, 1989) à proposer des théologies de la souffrance de Dieu, de sa non-puissance et de sa vulnérabilité. À ma connaissance, aucune théologie de la souffrance et de l'impuissance de Dieu n'a encore été développée du côté de l'islam.

4. N'y a-t-il pas que la mort qui soit fatale? Ce qui est certain, c'est que le mot fatum, arrêt du destin, qui dérive du verbe fari, parler (il s'agit d'une parole prédictive, d'un oracle), a donné naissance au substantif défunt-e et à l'adjectif feu, qui signifie défunt comme dans l'expression «feu mon ami ».

5. À la fin de 2004, le tsunami qui a ravagé une partie de l'Indonésie, du Sri Lanka et de quelques autres pays illustre bien ce que peut être une souffrance-malheur. En effet, ce sont des mouvements de plaques tectoniques qui sont à l'origine du malheur et de la souffrance qui accablent ces milliers de sinistrés. Ce malheur n'est donc pas un mal voulu, commis et actif, donc un mal de faute. Par ailleurs, il faut reconnaître que si une très grande partie de la population n'avait pas été obligée de vivre dans les zones côtières, car dépendante des seules ressources de la mer pour leur nourriture, leurs emplois et leurs revenus, le bilan des morts aurait été beaucoup moins lourd. De la même façon, si ces pays avaient été assez riches pour se doter d'un système de surveillance des tsunamis et des raz de marée, comme le Japon par exemple, les effets du tsunami auraient été beaucoup moins catastrophiques.

6. Un autre mot est employé ici: make'ôv, souffrance, qui provient de la racine $k^{\prime} v$, souffrir, comme l'assyrien $i k k i b u$, souffrance.

7. Qui dit deuil, dit douleur, souffrance, comme nous le rappelle l'origine du mot deuil qui provient du verbe latin dolore, souffrir. De manière semblable, le mot grec penthos, qui signifie à la fois deuil et douleur, dérive du verbe paschô, souffrir. 